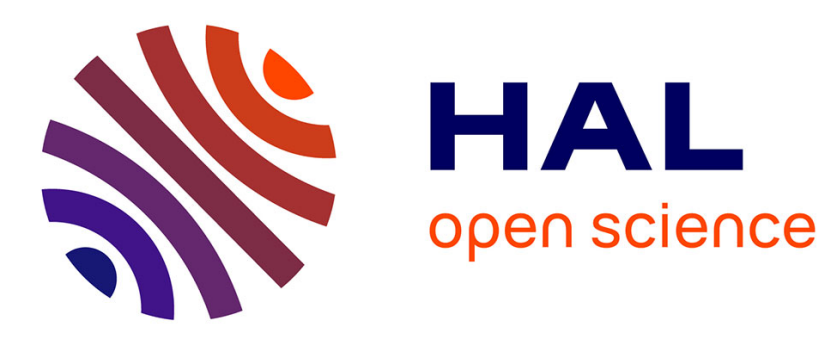

\title{
Time-suboptimal feedback design via linear matrix inequalities
}

\author{
Andrey Polyakov
}

\section{To cite this version:}

Andrey Polyakov. Time-suboptimal feedback design via linear matrix inequalities. Automation and Remote Control / Avtomatika i Telemekhanika, 2015, 76 (5), pp. 847-862. 10.1134/S0005117915050100 . hal-01215035

\section{HAL Id: hal-01215035 \\ https://inria.hal.science/hal-01215035}

Submitted on 16 Dec 2015

HAL is a multi-disciplinary open access archive for the deposit and dissemination of scientific research documents, whether they are published or not. The documents may come from teaching and research institutions in France or abroad, or from public or private research centers.
L'archive ouverte pluridisciplinaire HAL, est destinée au dépôt et à la diffusion de documents scientifiques de niveau recherche, publiés ou non, émanant des établissements d'enseignement et de recherche français ou étrangers, des laboratoires publics ou privés. 


\title{
Time-Suboptimal Feedback Design via Linear Matrix Inequalities
}

\author{
A. E. Polyakov \\ INRIA, Lille \\ e-mail: andrey.polyakov@inria.fr \\ Received MONTHEMBER XX, 2014
}

\begin{abstract}
In this paper, a time-optimal control problem is considered for plants represented by chains of integrators. A suboptimal solution obtained by using the implicit Lyapunov function approach is proposed in the form of continuous finite-time state feedback regulator. An algorithm for optimal tuning the parameters of the controller is formulated as a finite-dimensional semidefinite program. A robustness-oriented comparison of the optimal and suboptimal solutions in practical implementations of the proposed controller is performed via the numerical example of double integrator.
\end{abstract}

\section{INTRODUCTION}

In control practice it is often a challenge to design a controller which guarantees the minimal transient time. This problem is know as the time-optimal control problem; it typically arises in robotics, space applications, control of submarine and surface mobile systems. In spite of the long-standing and prolific history of the optimal control theory formulated as early as in 1950s and 1960s in the works by A.A. Fel'dbaum [1], L.S. Pontryagin at al. [2], R. Bellman [3], optimal control problems are still in the focus of active research [4-7].

The so-called bang-bang control is a classical discontinuous solution of the time-optimal control problem, which is based on the profound Fel'dbaum's $n$ interval theorem [1]. Traditionally, the corresponding control law is implemented as open-loop control (i.e., without feedback), except for low-dimensional cases which admit time-optimal control design by using sliding modes; e.g., see $[5,6]$. In practice, however, not only time-optimality of a control algorithm is of interest, but also other performance indices which are associated with the robustness and accuracy of the algorithm, its energy characteristics, etc. These indices are often conflicting, so that control design problems are sometimes reformulated in terms of quite hard multi-objective optimization. Finally, discontinuous time-optimal control laws are often impossible to implement because of physical peculiarities of the system $[8,9]$. In this respect, suboptimal controllers $[10,11]$ are of great practical importance. They are usually being designed under additional constraints on the class of admissible feedbacks (for instance, those leading to robust control laws) and other, not always constructive practice-driven requirements.

In this paper we consider the time-optimal control problem for a simplest plant represented by a chain of integrators, which is governed by a linear dynamic state feedback. In spite of the linear formulation of the problem, control design will be performed with the use of methods which are more popular in the nonlinear control theory. In particular, the nonlinear implicit control law proposed in this paper provides the closed-loop system with the so-called property of weighted homogeneity with negative exponent [12-15]. It is this property that guarantees robustness and finite-time stability, the latter being the ability for an asymptotically stable system to attain its equilibrium in finite time. On top of that, the ideas of weighted homogeneity are fundamental to 
the implicit Lyapunov function method [16,17], which is applied in this paper to suboptimal control design. To analyse stability of a system, this method suggests use of a Lyapunov function which is specified implicitly, e.g., in the form of an algebraic equation. Generally speaking, neither the stability analysis, nor the evaluation of the rate of convergence do require finding a solution of such an equation, since with the classical implicit function theorem [18], the corresponding conditions of the Lyapunov theorem can be checked by analyzing only the properties of this algebraic equation and the right-hand sides of the system. In the Russian-language control literature, similar ideas were proposed by V.I. Korobov [19] in the construction of the "controllability function," which is essentially a finite-time Lyapunov function of the closed-loop system.

The salient feature of combining the implicit Lyapunov function method with the theory of homogeneous system is that the algorithm for tuning the parameters of essentially nonlinear feedbacks [17] can be formalized in terms of linear matrix inequalities (LMIs) [20,21]. At present, LMIs are considered as the most efficient computational approach to linear control system design [20]; it allows for a considerable simplification of $H_{2} / H_{\infty}$-robust control design procedures [21]. Many other problems in control theory [20] can be reduced to solving semidefinite programs (SDPs), i.e., to the optimization of a (usually linear) cost subject to LMI constraints. In a number of works conducted by B.T. Polyak in the recent years, this approach was applied to the design of robust control for optimal rejection of exogenous disturbances in linear systems [21]. An extension of these ideas to nonlinear control plants can be found in [22]. In this paper, the nonlinear time-suboptimal design problem also reduces to solving a semidefinite program.

Notation. In the paper, the following notation will be used: $\mathbb{R}_{+}=\{x \in \mathbb{R}: x>0\}, \mathbb{R}_{-}=$ $\{x \in \mathbb{R}: x<0\}$, where $\mathbb{R}$ is the field of real numbers; $\operatorname{diag}\left\{\lambda_{i}\right\}_{i=1}^{n}$ is the diagonal matrix with the entries $\lambda_{i}$ on the main diagonal; the continuous function $\sigma: \mathbb{R}_{+} \rightarrow \mathbb{R}_{+}$belongs to the class $\mathcal{K}$ if it is monotonically increasing and $\sigma(s) \rightarrow 0^{+}$as $s \rightarrow 0^{+}$; if this function is not bounded as $s \rightarrow+\infty$, we say that it belongs to the class $\mathcal{K}_{\infty}$; a continuous function $\beta: \mathbb{R}_{+} \times \mathbb{R}_{+} \rightarrow \mathbb{R}_{+}$belongs to the class $\mathcal{K} \mathcal{L}$ if $\beta(\cdot, t) \in \mathcal{K}_{\infty}$ for any fixed $t \in \mathbb{R}_{+}$, and $\beta(s, \cdot)$ is monotonically decreasing for any fixed $s \in \mathbb{R}_{+}$; for a matrix $P \in \mathbb{R}^{n \times n}$ with real spectrum, its minmal and maximal eigenvalues are denoted by $\lambda_{\min }(P)$ and $\lambda_{\max }(P)$, respectively; for a symmetric matrix $P \in \mathbb{R}^{n \times n}$, the inequality $P>0$ $(P \geq 0, P<0, P \leq 0)$ is understood as positive definiteness (respectively, positive semidefiniteness, negative definiteness, negative semidefiniteness); $\mathbb{C}^{k}$ is the space of functions having derivatives up to order $k$ inclusive; $\mathcal{L}$ is the space of Lebesgue integrable functions; $\mathcal{L}^{\infty}$ is the space of measurable, almost everywhere bounded functions.

\section{STATEMENT OF THE PROBLEM}

In this paper we analyze a single-input control plant represented by a chain of $n$ integrators:

$$
\dot{x}(t)=A x(t)+b u(t), \quad t \in[0, T], \quad x(0)=x_{0} \in \mathbb{R}^{n},
$$

where $T \in R_{+}$is a finite time instant, $x \in \mathbb{R}^{n}$ is the state vecror, $u \in \mathbb{R}$ is the control input, and $A=\left(\begin{array}{cc}0 & I_{n-1} \\ 0 & 0\end{array}\right), b=(0, \ldots, 0,1)^{\top} \in \mathbb{R}^{n}$. The importance of this model is stipulated by numerous mechanical and electromechanical applications, see [5, 23,24].

Let us consider the time optimal control problem in the classical formulation:

$$
T \rightarrow \min
$$


subject to the constraints

$$
\begin{aligned}
& u(\cdot) \in \mathcal{U}=\left\{u(\cdot) \in \mathcal{L}_{(0, T)}:|u(t)| \leq u_{0}, t \in[0, T]\right\}, \\
& x(\cdot) \in \mathbb{C}_{[0, T]}:\left\{\begin{array}{l}
\dot{x}(t)=A x(t)+b u(t), t \in(0, T), \\
x(0)=x_{0}, \quad x(T)=0,
\end{array}\right.
\end{aligned}
$$

where $x_{0} \in \mathbb{R}^{n}$ and $u_{0} \in \mathbb{R}_{+}$are given.

By the Feldbaum theorem [1], the optimal solution is provided by the so-called "bang-bang" control, which is a piece-wise constant function $u_{\text {opt }}(t) \in\left\{-u_{0}, u_{0}\right\}, t \in(0, T)$, with $n-1$ discontinuities. In this paper we will be interested in solving problem (2), (3), subject to certain additional constraints. First, admissible input signals are assumed to be smooth, $u(\cdot) \in \mathbb{C}_{(0, T)}^{\infty}$. This restriction is natural in many problems encountered in practice, $[25,26]$. This condition is often adopted in order to guarantee that the control law be implementable [27]. Linear control laws can be designed close to optimal ones [11]; hence, we additionally limit ourselves to the class of smooth, linear dynamical state controllers:

$$
u(\cdot)=w^{\top}(\cdot) x(\cdot) \in \mathcal{U}, \quad w=\left(w_{1}, w_{2}, \ldots, w_{n}\right)^{\top}, \quad w_{i}(\cdot) \in \mathbb{C}_{(0, T)}^{\infty} .
$$

"Rubustification" of optimal controllers is equally important, [5-7]. This requirement unavoidably leads to feedback control algorithms. In this paper we are interested in nonlinear continuous robust state feedback control laws $\left(u(t)=u_{f}(x(t)), t>0, u_{f}: \mathbb{R}^{n} \rightarrow \mathbb{R}\right)$ such that they globally stabilize system (1) at the origin for all $t>0$ and, moreover, provide feasible solutions to the optimization problem (2)-(4). It is this class of feedback laws over which we will be searching for a suboptimal solution of the time-optimal control problem (2)-(4) with given $x_{0} \in \mathbb{R}^{n}$.

Yet another limitation (strictly speaking, not an intrinsic one) in the problem analyzed in this paper is the necessity of using the apparatus of linear matrix inequalities. Actually, this requirement may be thought of as the most important one, since control practice is always targeted at finding simplest and most efficient design solutions. Moreover, with this technique, the infinite-dimensional optimization problem (2)-(4) will be reduced to a finite-dimensional problem.

Finally, note that the time optimal control problem with terminal state $x(T)=x_{1}:=(\xi, 0, \ldots, 0)^{\top} \in$ $\mathbb{R}^{n}, \xi \neq 0$ can be easily reduced to problem (2)-(4) by change of variables $\tilde{x}=x-x_{1}$.

\section{AUXILIARY CONCEPTS AND RESULTS}

\subsection{Finite-Time Stability}

Control design problems under constraints (3) are usually referred to as finite-time control design. Problems of such type are traditionally considered over finite time horizon, while the issues of stability and robustness are kept aside, since the solution is usually turns out to be a certain openloop (feedforward) control law. Instead, in this paper we are interested in finite-time regulators, which specify a stabilizing feedback control and, on top of asymptotic stability of the closed loop system, guarantee attainment of the equilibrium in finite time [28-32].

Let us consider a Cauchy problem for the ordinary differential equation

$$
\dot{x}(t)=f(t, x(t)), \quad t \in \mathbb{R}, \quad x\left(t_{0}\right)=x_{0},
$$

where $t_{0}$ is a number, $x \in \mathbb{R}^{n}$ is the state vector, $f: \mathbb{R} \times \mathbb{R}^{n} \rightarrow \mathbb{R}^{n}$ is a nonlinear, continuous vector-valued function, which is locally Lipschitz in $x$ everywhere except for the origin.

Let the origin be an equilibrium point of system (5), i.e., $f(t, 0)=0$. 
Definition 1. The zero solution of system (5) is said to be finite-time stable if it is Lyapunov stable and there exists a function $T: \mathbb{R} \times \mathbb{R}^{n} \rightarrow \mathbb{R}_{+}$such that $\lim _{t \rightarrow t_{0}+T\left(t_{0}, x_{0}\right)} x\left(t, t_{0}, x_{0}\right)=0$ for all $x_{0} \in \mathcal{V} \subseteq \mathbb{R}^{n}$, where $x\left(t, t_{0}, x_{0}\right)$ is the solution of the Cauchy problem (5), and $\mathcal{V}$ is a neighborhood of the origin, which, generally speaking, depends on $t_{0}$.

The definition of stability formulated above almost coincides with the definition of asymptotic stability, with the only difference that the limit is considered as $t$ tends to a certain finite number. Importantly, the requirement of the Lyapunov stability in Definition 1 obviously ensures $x\left(t, t_{0}, x_{0}\right)=0$ for all $t \geq t_{0}+T\left(t_{0}, x_{0}\right)$. In other words, any finite-time stable system is asymptotically stable.

The function $T$ in Definition 1 is referred to as settling-time function of system (5). If the set $\mathcal{V}$ coincides with $\mathbb{R}^{n}$, the zero solution of system (5) becomes finite-time stable in the large, or globally finite-time stable.

Following the ideas of the stability theory, a natural definition of the uniform finite-time stability can be formulated. It is important to note that uniformity is to be considered with respect to both time and the space $\mathbb{R}^{n}$, since inherent to the finite-time stability is the dependence of the settlingtime function on both the initial time instant and the initial state. In particular, if the function of ("uniform") settling time $T(\cdot)=\sup _{t_{0} \in \mathbb{R}} T\left(t_{0}, \cdot\right)$ is locally bounded in a certain $t_{0}$-independent neighborhood $\mathcal{V}$ of the origin, the finite-time stable system is said to be uniformly finite-time stable $[30,32]$. As of today, the most comprehensive survey of the results in finite-time stability can be found in [32].

A simple example of a uniformly finite-time stable system is given by a scalar control plant embraced by the negative relay state feedback: $\dot{x}(t)=-\operatorname{sign}[x(t)], x\left(t_{0}\right)=x_{0} \in \mathbb{R}$. In this case, the settling-time function has the form $T\left(x_{0}\right)=\left|x_{0}\right|$.

\subsection{Weighted Homogenous Systems}

Homogeneity is a property of an object (say, a vector field) to retain its characteristics under certain stretcing/shrinkage. This property turns out to be extremely useful in many areas such as analysis of nonlinear ordinary differential equations $[12,33-35]$ and finite-time stability $[14,30,36]$, robustness of nonlinear control systems [37-39], etc.

Let us consider a positive vector of weights $r=\left(r_{1}, \ldots, r_{n}\right)^{\top} \in \mathbb{R}_{+}^{n}$ and a so-called dilation matrix $D_{r}(\lambda)=\operatorname{diag}\left\{\lambda^{r_{i}}\right\}_{i=1}^{n}, \quad \lambda \in \mathbb{R}_{+}$. Clearly, for $x=\left(x_{1}, \ldots, x_{n}\right)^{\top} \in \mathbb{R}^{n}$ we have $D_{r}(\lambda) x=$ $\left(\lambda^{r_{1}} x_{1}, \ldots, \lambda^{r_{i}} x_{i}, \ldots, \lambda^{r_{n}} x_{n}\right)^{\top} \in \mathbb{R}^{n}$.

Definition 2 ([12]). A function $g: \mathbb{R}^{n} \rightarrow \mathbb{R}$ (respectively, a vector field $f: \mathbb{R}^{n} \rightarrow \mathbb{R}^{n}$ ) is referred to as $r$-homogeneous with degree $m \in \mathbb{R}$, if the equality $g\left(D_{r}(\lambda) x\right)=\lambda^{m} g(x)$ (respectively, $\left.f\left(D_{r}(\lambda) x\right)=\lambda^{m} D_{r}(\lambda) f(x)\right)$ holds for any $\lambda \in \mathbb{R}_{+}$and any $x \in \mathbb{R}^{n}$.

Functions that fall under Definition 2 will be also referred to as weighted homogeneous.

Homogeneous and weighted homogeneous system are often encountered in control theory. First of all note that linear systems are obviously homogeneous of degree zero with $D_{r}(\lambda)=\lambda I_{n}$. Design of nonlinear algorithms for control and state identification which are based on the homogeneity property is discussed in $[14,17,30,36]$.

Following Definition 2, homogeneity can be detected by checking just the right-hand sides of ordinary differential equations. By knowing the degree of homogeneity, finite-time stability of the system can be established via the theorem below. 
Theorem 1 ([40], Theorem 5.8 and Property 5.4). If the vector field $f: \mathbb{R}^{n} \rightarrow \mathbb{R}^{n}$ is defined in $\mathbb{R}^{n}$, continuous, and $r$-homogeneous with negative degree $m \in \mathbb{R}_{-}$, then the asymptotically stable zero solution of the system $\dot{x}(t)=f(x(t)), t \in \mathbb{R}$, is globally finite-time stable.

This result remains valid for homogeneous differential inclusions and generalized evolutionary equations in Banach spaces, $[15,30,36]$.

It is important to note that, on top of detecting finite-time stability, the homogeneity method facilitates qualitative analysis of robustness.

By robustness of a control system we usually mean its stability (in one or another sense) against exogenous disturbances and inaccuracies in the parameters of the model. In this paper, robustness is considered in the sense of input-to-state stability [41,42].

Consider a disturbed system

$$
\dot{x}(t)=f(x(t), d(t)), \quad t>0, \quad x(0)=x_{0} \in \mathbb{R}^{n},
$$

where $x(t) \in \mathbb{R}^{n}$ is the state vector, $d(t)=\left(d_{1}(t), \ldots, d_{p}(t)\right)^{\top} \in \mathbb{R}^{p}, d_{i}(\cdot) \in \mathcal{L}_{\infty}$ is the vector of exogenous disturbances, and $f: \mathbb{R}^{n} \times \mathbb{R}^{p} \rightarrow \mathbb{R}^{n}$ is a (generally speaking discontinuous [15]) vector field.

Definition 3 ([41]). System (6) is referred to as input-to-state stable (ISS), if, for all $x_{0} \in \mathbb{R}^{n}$ and all $d \in \mathcal{L}^{\infty}$, the ineuality $\left\|x\left(t, x_{0}\right)\right\| \leq \beta\left(\left\|x_{0}\right\|, t\right)+\gamma(\underset{\tau \in[0, t)}{\operatorname{ess} \sup }\|d(\tau)\|)$ is valid for all $t \in \mathbb{R}_{+}$ over any solution $x\left(t, x_{0}\right)$ of the Cauchy problem (6), where $\beta \in \mathcal{K} \mathcal{L}$ and $\gamma \in \mathcal{K}$.

A weaker notion of robustness is introduced as follows.

Definition 4 ([41]). System (6) is said to be integral input-to-state stable (iISS), if for all $x_{0} \in \mathbb{R}^{n}$ and all $d \in \mathcal{L}^{\infty}$ the inequality $\alpha\left(\left\|x\left(t, x_{0}\right)\right\|\right) \leq \beta\left(\left\|x_{0}\right\|, t\right)+\int_{0}^{t} \gamma(\|d(\tau)\|) d \tau$ holds for all $t \geq 0$ over any solution $x\left(t, x_{0}\right)$ of system (6) for some $\alpha \in \mathcal{K}_{\infty}, \beta \in \mathcal{K} \mathcal{L}$, and $\gamma \in \mathcal{K}$.

The theorem below establishes a link between robust stability and homogeneity.

Theorem 2 ([39]). Let the following conditions be satisfied:

1) the zero solution of the disturbance-free $(d=0)$ system $(6)$ is asymptotically stable;

2) the disturbance-free vector field $f(\cdot, 0): \mathbb{R}^{n} \rightarrow \mathbb{R}^{n}$ is $r$-homogeneous with degree $m \geq-\min _{1 \leq i \leq n} r_{i}$;

3) there exists $\tilde{\mathbf{r}}=\left(\tilde{r}_{1}, \ldots, \tilde{r}_{p}\right)^{\top} \in \mathbb{R}^{p}, \tilde{r}_{i} \geq 0$, such that $f\left(D_{r}(\lambda) x, D_{\tilde{r}}(\lambda) \mathrm{d}\right)=\lambda^{m} D_{r}(\lambda) f(x, \mathrm{~d})$ for all $x \in \mathbb{R}^{n}, d \in \mathbb{R}^{p}$, and $\lambda \in \mathbb{R}_{+}$.

Then system (6) is input-to-state stable if $\tilde{r}_{\min }>0$, and it is integral input-to-state stable if $\tilde{r}_{\min }=0$ and $m \leq 0$, where it is denoted $\tilde{r}_{\min }=\min _{1 \leq j \leq p} \tilde{r}_{j}$.

From these results it is seen that robust and finite-time stability of homogeneous systems can be expressed in terms of a simple function of the degree of homogeneity and the vector of weights. These properties can be easily checked via elementary algebraic manipulations.

\subsection{Implicit Lyapunov Functions}

The implicit Lyapunov function method assumes an implicit specification of a function, for instance, in the form of an algebraic equation $Q(V, x)=0$. The theorem below is an extension of the known result in [16] to the analysis of finite-time stability. 
Theorem 3 ([17]). Assume there exists a continuous function

$$
\begin{gathered}
Q: \mathbb{R}_{+} \times \mathbb{R}^{n} \rightarrow \mathbb{R} \\
(V, x) \mapsto Q(V, x)
\end{gathered}
$$

with the following properties:

C1) $Q$ is continuously differentiable for all $V \in \mathbb{R}_{+}$and all $x \in \mathbb{R}^{n} \backslash\{0\}$;

C2) for every $x \in \mathbb{R}^{n} \backslash\{0\}$ there exists $V \in \mathbb{R}_{+}$such that $Q(V, x)=0$;

C3) $\lim _{\substack{x \rightarrow 0 \\(V, x) \in \Omega}} V=0^{+}, \lim _{\substack{V \rightarrow 0^{+} \\(V, x) \in \Omega}}\|x\|=0, \lim _{\substack{\|x\| \rightarrow \infty \\(V, x) \in \Omega}} V=+\infty$, where $\Omega=\left\{(V, x) \in \mathbb{R}^{n+1}: Q(V, x)=0\right\}$;

C4) the inequality $-\infty<\frac{\partial Q(V, x)}{\partial V}<0$ holds for all $V \in \mathbb{R}_{+}$and all $x \in \mathbb{R}^{n} \backslash\{0\}$;

C5) for all $t \in \mathbb{R}_{+}$and all $(V, x) \in \Omega$ the following inequality holds: $\frac{\partial Q(V, x)}{\partial x} f(t, x) \leq c V^{1-\mu} \frac{\partial Q(V, x)}{\partial V}$, where $c>0$ and $0<\mu \leq 1$ are some numbers.

Then the zero solution of system (5) is globally finite-time stable and the following estimate is valid for the settling-time function: $T\left(x_{0}\right) \leq \frac{V_{0}^{\mu}}{c \mu}$, where $V_{0} \in \mathbb{R}_{+}: Q\left(V_{0}, x_{0}\right)=0$.

Conditions $C 1-C_{4}$ of Theorem 3 guarantee the uniqueness, positive-definiteness, smoothness (outside the origin), and radial unboundedness of the solution of $Q(V, x)=0$ with respect to $V$. By the classical implicit function theorem (e.g., see [18]), the partial derivative of the function $V: \mathbb{R}^{n} \rightarrow$ $\mathbb{R}$ can be computed via the formula $\frac{\partial V}{\partial x}=-\left[\frac{\partial Q}{\partial V}\right]^{-1} \frac{\partial Q}{\partial x}$. Hence, the condition $\frac{\partial Q(V, x)}{\partial x} f(t, x)<0$ ensures the negative definiteness of the total derivative of $V$, and condition $C 5$ guarantees the finite-time stability of system (5) due to the inequality $\dot{V}(x(t)) \leq-c V^{1-\mu}(x(t))$.

Introduce now the explicit Lyapunov function as

$$
Q(V, x):=x^{\top} D_{r}\left(V^{-1}\right) P D_{r}\left(V^{-1}\right) x-1,
$$

where $D_{r}(\lambda)$ is the dilation matrix of the form $D_{r}(\lambda)=\operatorname{diag}\left\{\lambda^{r_{i}}\right\}_{i=1}^{n}, r=\left(r_{1}, \ldots, r_{n}\right)^{\top} \in \mathbb{R}_{+}^{n}$, and $P \in \mathbb{R}^{n \times n}$ is a symmetric positive definite matrix.

This function can be thought of as a counterpart of a quadratic Lyapunov function for $r$ homogeneous functions. Indeed, for any fixed $V=$ const, the set $\left\{x \in \mathbb{R}^{n}: Q(V, x)=0\right\}$ is the surface of an ellipsoid in $\mathbb{R}^{n}$. For $r=(0,5, \ldots, 0,5)^{\top}$, the equation $Q(V, x)=0$ possesses the unique solution $V=x^{\top} P x$.

Corollary 1. Let a vector field $f: \mathbb{R}^{n} \rightarrow \mathbb{R}^{n}$ be r-homogeneous of degree $m$, with the weight vector $r=\left(r_{1}, r_{2}, \ldots, r_{n}\right)^{\top} \in \mathbb{R}_{+}^{n}$. If there exists a matrix $P \in \mathbb{R}^{n \times n}$ which satisfies the inequalities

$$
\begin{gathered}
\operatorname{diag}(r) P+P \operatorname{diag}(r)>0, \quad P>0, \\
z^{\top} P f(z)+f^{\top}(z) P z<0 \text { for } z \in \mathbb{R}^{n}: z^{\top} P z=1,
\end{gathered}
$$

then the zero solution of system $\dot{x}(t)=f(x(t)), t>0$, is asymptotically stable, and the function $Q$ defined by (7) is an implicit Lyapunov function for the system. Moreover, for $m<0$ the system is finite-time stable.

Proof. I. The function $Q(V, x)$ defined by Ineq. (7) satisfies Conditions $C 1-C 4$ of Theorem 3. Indeed, it is continuously differentiable for all $V \in \mathbb{R}_{+}$and all $x \in \mathbb{R}^{n}$. Since $P>0$, the chain of inequalities $\frac{\lambda_{\min }(P)\|x\|^{2}}{\max \left\{V^{1+(n-1) \mu}, V\right\}} \leq Q(V, x)+1 \leq \frac{\lambda_{\max }(P)\|x\|^{2}}{\min \left\{V^{1+(n-1) \mu}, V\right\}}$ guarantees that for any $x \in \mathbb{R}^{n} \backslash\{0\}$ there exist $V^{-} \in \mathbb{R}_{+}$and $V^{+} \in \mathbb{R}_{+}$such that $Q\left(V^{-}, x\right)<0<Q\left(V^{+}, x\right)$, while the continuity of the function $Q$ implies the existence of $V \in \mathbb{R}^{+}$such that $Q(V, x)=0$. 
Moreover, for $Q(V, x)=0$, the same chain of inequalities ensures $\frac{\min \left\{V^{1+(n-1) \mu}, V\right\}}{\lambda_{\max }(P)} \leq\|x\|^{2} \leq$ $\frac{\max \left\{V^{1+(n-1) \mu}, V\right\}}{\lambda_{\min }(P)}$; hence, Condition $C 3$ of Theorem 3 is also fulfilled.

Since $\frac{\partial Q}{\partial V}=-V^{-1} x^{\top} D_{\mu}\left(V^{-1}\right)(\operatorname{diag}(r) P+P \operatorname{diag}(r)) D_{\mu}\left(V^{-1}\right) x$, Ineq. (8) implies $\frac{\partial Q}{\partial V}<0$ for all $V \in \mathbb{R}_{+}$and all $x \in \mathbb{R}^{n} \backslash\{0\}$.

By the implicit function theorem, there exists a unique positive definite, smooth (outside the origin), and radially unbounded solution $V: \mathbb{R}^{n} \rightarrow \mathbb{R}$ of the equation $Q(V, x)=0$, which can be continued to the origin as $V(0)=0$ and $Q(V(x), x)=0$ for $x \in \mathbb{R}^{n} \backslash\{0\}$.

II. Let us compute the total derivative of the function $V$ along the trajectories of the system:

$$
\dot{V}(x)=-\left[\frac{\partial Q}{\partial V}\right]^{-1} \frac{\partial Q}{\partial x} f(x)=-\left[\frac{\partial Q}{\partial V}\right]^{-1}\left(z^{\top} P D_{r}\left(V^{-1}\right) f(x)+f(x)^{\top} D_{r}\left(V^{-1}\right) P z\right),
$$

where $x \in \mathbb{R}^{n} \backslash\{0\} \quad z=D_{r}\left(V^{-1}\right) x$. The vector field $f$ is homogeneous, i.e., $\lambda^{-m} D_{r}^{-1}(\lambda) f\left(D_{r}(\lambda) x\right)=$ $f(x)$ for all $\lambda \in \mathbb{R}_{+}$and all $x \in \mathbb{R}^{n}$, where $m \in \mathbb{R}$ is the degree of homogeneity. We then have

$$
\dot{V}(x)=-\left[\frac{\partial Q}{\partial V}\right]^{-1} V^{-m}\left(z^{\top} P y+y^{\top} P z\right)=V^{1-m} \frac{z^{\top} P f(z)+f(z)^{\top} P z}{z^{\top}(\operatorname{diag}(r) P+P \operatorname{diag}(r)) z} .
$$

Since $Q(V, x)=0$, relations $z^{\top} P z=1$ and (9) imply $\dot{V}(x)<0$ for $x \neq 0$.

\section{CONTROL DESIGN}

\subsection{Design of Homogenous Finite-Time Controllers}

Design of finite-time controllers will be accomplished by using the concept of $r$-homogeneity of the closed-loop system. In that case, for some $r \in \mathbb{R}_{+}^{n}$ and $\mu \in \mathbb{R}_{+}$, the equality $A D_{r}(\lambda) x+$ $b u\left(D_{r}(\lambda) x\right)=\lambda^{-\mu} D_{r}(\lambda)(A x+b u(x))$ is to be satisfied for all $\lambda \in \mathbb{R}_{+}$and $x \in \mathbb{R}^{n}$. Hence, keeping in mind the structure of the matrix $A$ and vector $b$, we obtain explicit bounds on the the components of the weight vector $r \in \mathbb{R}_{+}^{n}: r_{i}=r_{i+1}+\mu, i=1,2, \ldots, n-1$, and on the degree of homogeneity of the control function: $u\left(D_{r}(\lambda) x\right)=\lambda^{r_{n}-\mu} u(x)$.

By taking $r_{n}=1$, we obtain $r=(1+(n-1) \mu, 1+(n-2) \mu, \ldots, 1)^{\top} \in \mathbb{R}_{+}^{n}$, where $\mu \in(0,1]$. The theorem below is a simple extension of the results in [43] and [17].

Theorem 4. Assume that $X \in \mathbb{R}^{n \times n}$ and $y \in \mathbb{R}^{1 \times n}$ satisfy the linear matrix inequalities

$$
\left\{\begin{array}{c}
A X+X A^{\top}+b y+y^{\top} b^{\top}+\operatorname{diag}(r) X+X \operatorname{diag}(r)=0, \\
X \operatorname{diag}(r)+\operatorname{diag}(r) X>0, \quad X>0,
\end{array}\right.
$$

for some fixed $\mu \in(0,1]$.

Then system (1) embraced by the feedback

$$
u(V, x)=V^{1-\mu} k D_{r}\left(V^{-1}\right) x
$$

with $k=y X^{-1}, V \in \mathbb{R}_{+}$and $Q(V, x)=0$, where $Q(V, x)$ is of the form (7) with $P=X^{-1}$, is globally uniformly finite-time stable with the settling-time function

$$
T_{0}\left(x_{0}\right)=\frac{V_{0}^{\mu}}{\mu},
$$

where $V_{0} \in \mathbb{R}_{+}: Q\left(V_{0}, x_{0}\right)=0$. 
Proof. The fulfillment of conditions $C 1-C_{4}$ of Theorem 3 for the implicit function $Q$ specified by (7) was established in the proof of Corollary 1 . It now remains to check the satisfaction of Condition C5. Since

$$
\frac{\partial Q}{\partial x}(A x+b u(x))=2 x^{\top} D_{r}\left(V^{-1}\right) P D_{r}\left(V^{-1}\right)(A x+b u(x)),
$$

equalities $D_{r}\left(V^{-1}\right) A D_{r}^{-1}\left(V^{-1}\right)=V^{-\mu} A$ and $D_{r}\left(V^{-1}\right) b u(x)=V^{-\mu} b k D_{r}\left(V^{-1}\right) x$ imply the relations $\frac{\partial Q}{\partial x}(A x+b u(x))=V^{-\mu} x^{\top} D_{r}\left(V^{-1}\right)\left(P(A+b k)+(A+b k)^{\top} P\right) D_{\mu}\left(V^{-1}\right) x$ and

$$
\dot{V}(x)=-\left[\frac{\partial Q}{\partial V}\right]^{-1} \frac{\partial Q}{\partial x}(A x+b u)=\frac{x^{\top} D_{r}\left(V^{-1}\right)\left(P(A+b k)+(A+b k)^{\top} P\right) D_{r}\left(V^{-1}\right) x}{x^{\top} D_{r}\left(V^{-1}\right)(\operatorname{diag}(r) P+P \operatorname{diag}(r)) D_{r}\left(V^{-1}\right) x} V^{1-\mu}=-V^{1-\mu}(x) .
$$

The last equality guarantees the finite-time stability of the closed-loop system (1), (11), and representation (12) of the settling-time function.

The control law (11) represents an implicitly defined nonlinear controller whose properties are formulated in the corollary below.

Corollary 2. The implicitly defined nonlinear state feedback controller $\tilde{u}(x)=u(V(x), x)$ with $V: \mathbb{R}^{n} \rightarrow \mathbb{R}$ such that $Q(V(x), x)=0$ for all $x \in \mathbb{R}^{n} \backslash\{0\}$, has the following properties:

1) continuity in $\mathbb{R}^{n}$ for $\mu \in(0,1)$;

2) discontinuity only at the origin and global boundedness in $\mathbb{R}^{n}$ for $\mu=1$;

3) $r$-homogeneity with degree $1-\mu$ for $\mu \in[0,1]$, i.e., $\tilde{u}\left(D_{r}(\lambda) x\right)=\lambda^{1-\mu} \tilde{u}(x)$ for all $x \in \mathbb{R}^{n}$ and $\lambda \in \mathbb{R}_{+}$.

Proof. 1. The function $V: \mathbb{R}^{n} \rightarrow \mathbb{R}$ explicitly specified by the equation $Q(V, x)=0$ is continuously differentiable for all $x \in \mathbb{R}^{n} \backslash\{0\}$. Moreover, $V(x) \rightarrow 0$ as $\|x\| \rightarrow 0$. Hence, the only possible point of discontinuity of the function $\tilde{u}$ is the origin. For $Q(V(x), x)=0$ we obtain

$$
\begin{gathered}
\tilde{u}^{2}(x)=V^{2-2 \mu}(x) x^{\top} T D_{r}\left(V^{-1}(x)\right) k^{\top} k D_{r}\left(V^{-1}(x)\right) x \leq \\
\leq V^{2-2 \mu}(x) \lambda_{\max }\left(k^{\top} k\right) x^{\top} D_{r}\left(V^{-1}(x)\right) D_{r}\left(V^{-1}(x)\right) x \leq \\
\leq \frac{V^{2-2 \mu}(x) \lambda_{\max }\left(k k^{\top}\right) x^{\top} D_{r}\left(V^{-1}(x)\right) P D_{r}\left(V^{-1}(x)\right) x}{\lambda_{\min }(P)}=V^{2-2 \mu}(x) \frac{\lambda_{\max }\left(k^{\top} k\right)}{\lambda_{\min }(P)} .
\end{gathered}
$$

Obviously, we have $\tilde{u}^{2}(x) \rightarrow 0$ as $\|x\| \rightarrow 0$ and $\mu \in(0,1)$, i.e., $\tilde{u}$ is a continuous function.

2. For $\mu=1$ we have $\tilde{u}^{2}(x) \leq \frac{\lambda_{\max }\left(k^{\top} k\right)}{\lambda_{\min }(P)}$ for all $x \in \mathbb{R}^{n}$. To prove the discontinuity of the control input in this situation, it suffices to consider $\tilde{x}=\left(0, \ldots, 0, x_{n}\right)^{\top} \in \mathbb{R}^{n}$. In this case we have $V(\tilde{x})=\sqrt{b^{\top} P b}\left|x_{n}\right|$ and $u(\tilde{x})=\frac{k b}{\sqrt{b^{\top} P b}} \operatorname{sign}\left[x_{n}\right]$.

3. From $Q\left(V, D_{r}(\lambda) x\right)=Q\left(\lambda^{-1} V, x\right)$ we obtain $V\left(D_{r}(\lambda) x\right)=\lambda V(x)$ and $\tilde{u}\left(D_{r}(\lambda) x\right)=\lambda^{1-\mu} \tilde{u}(x)$ by the equality $V^{1-\mu}\left(D_{r}(\lambda) x\right) k D_{r}\left(V^{-1}\left(D_{r}(\lambda) x\right)\right) D_{r}(\lambda) x=\lambda^{1-\mu} V^{1-\mu}(x) k D_{r}\left(\lambda^{-1} V^{-1}(x)\right) D_{r}(\lambda) x$.

Note that the $r$-homogeneity property of the controller $\tilde{u}$ clearly implies the $r$-homogeneity of the closed-loop system. Theorem 2 ensures the robustness of the system against various disturbances, e.g., such as measurement noises. Indeed, if $\dot{x}=f(x)$ is weighted homogeneous, then $\dot{x}=f(x+d)$ satisfies Theorem 2.

One of the attractive properties of the finite-time control design scheme described above is its simplicity in tuning the parameters on the basis of linear matrix inequalities; these can be solved by using various available software, e.g., MATLAB. 
Proposition 1. Inequalities (10) are feasible for all $\mu>0$.

Proof. Consider the following component-wise representation of the matrix $X$ and vector $y$ :

$$
\begin{gathered}
X=\left(\begin{array}{ccccc}
x_{11} & x_{12} & \ldots & x_{1 n-1} & x_{1 n} \\
x_{12} & x_{22} & \ldots & x_{2 n-1} & x_{2 n-1} \\
\ldots & \ldots & \ldots & \ldots & \ldots \\
x_{1 n-1} & x_{2 n-1} & \ldots & x_{n-1 n-1} & x_{n-1 n} \\
x_{1 n} & x_{1 n-1} & \ldots & x_{n-1 n} & x_{n n}
\end{array}\right), x_{i j} \in \mathbb{R}, \quad i, j=1,2, \ldots, n ; \\
y=\left(\begin{array}{lllll}
y_{1} & y_{2} & \ldots & y_{n-1} & y_{n}
\end{array}\right), \quad y_{i} \in \mathbb{R}, \quad i=1,2, \ldots, n .
\end{gathered}
$$

The algebraic equation in system (10) can be written down in the following form:

$$
\begin{aligned}
2 x_{i+1}+2[1+\mu(n-i)] x_{i i} & =0, \quad i=1,2, \ldots, n-1, \\
x_{i+1 j}+x_{i j+1}+[2+\mu(2 n-i-j)] x_{i j} & =0, \quad j>i=1,2, \ldots, n-1, \\
x_{i+1 n}+[2+\mu(n-i)] x_{i n}+y_{i} & =0, \quad i=1,2, \ldots, n-1, \\
2 x_{n n}+2 y_{n} & =0 .
\end{aligned}
$$

Let $\mathbf{X}_{\left(i_{1}: i_{2} ; j_{1}: j_{2}\right)}$ denote the block of the matrix $X$ composed of the entries $x_{i j}$ satisfying $i=$ $i_{1}, i_{1}+1, \ldots, i_{2}$ and $j=j_{1}, j_{1}+1, \ldots, j_{2}$ for $i_{1} \leq i_{2}$ and $j_{1} \leq j_{2}$.

Denote next $H_{i}=\operatorname{diag}\{1+\mu(n-1), 1+\mu(n-2), \ldots, 1+\mu(n-i)\}$ and $Z=X \operatorname{diag}(r)+\operatorname{diag}(r) X$. Let $\mathbf{Z}_{\left(i_{1}: i_{2} ; j_{1}: j_{2}\right)}$ be the block of the matrix $Z$ composed similarly to the block $\mathbf{X}_{\left(i_{1}: i_{2} ; j_{1}: j_{2}\right)}$. Clearly, we have

$$
\mathbf{Z}_{(1: 1 ; 1: 1)}=2[1+\mu(k-1)] X_{(1: 1 ; 1: 1)}, \quad \mathbf{Z}_{(1: i ; 1: i)}=\left(\begin{array}{cc}
\mathbf{Z}_{(1: i-1 ; 1: i-1)} & \mathbf{X}_{(1: i-1 ; i: i)} H_{i} \\
\mathbf{X}_{(1: i-1 ; i: i)}^{\top} H_{i} & 2[1+\mu(k-i)] x_{i i}
\end{array}\right) .
$$

Let us construct a solution of (13)-(16) such that the conditions $X>0$ and $Z>0$ be satisfied. To this end, we use the method of mathematical induction.

Basis. Let $x_{11}=\alpha_{1}$, where $\alpha_{1}>0$ is an arbitrary positive number. From Eq. (13) we obtain $x_{12}=-\alpha_{1}[1+\mu(n-1)] x_{11}$. Noting that $x_{11}=\mathbf{X}_{(1: 1 ; 1: 1)}>0$ and $\mathbf{Z}_{(1: 1 ; 1: 1)}>0$, and taking $x_{22}=\alpha_{2}$, we can guarantee $\mathbf{X}_{(1: 2 ; 1: 2)}>0$ and $\mathbf{Z}_{(1: 2 ; 1: 2)}>0$ for sufficiently large $\alpha_{2}>0$.

Inductive step. Assume that for some $\tilde{k}<n$, the matrices $\mathbf{X}_{(1: \tilde{k} ; 1: \tilde{k})}>0$ and $\mathbf{Z}_{(1: \tilde{k} ; 1: \tilde{k})}>0$ are composed in such a way that $x_{i i}=\alpha_{i} \in \mathbb{R}_{+}$. From Eq. (13) we then have $x_{\tilde{k} \tilde{k}+1}=-\alpha_{\tilde{k}}[1+\mu(n-\tilde{k})]$, and using Eq. (14) we find $x_{i \tilde{k}+1}=-\left(x_{i+1} \tilde{k}+[2+\mu(2 n-i-\tilde{k})] x_{i \tilde{k}}\right), i=1,2 \ldots, \tilde{k}-1$.

Noting that $\mathbf{X}_{(1: \tilde{k} ; 1: \tilde{k})}>0$ and $\mathbf{Z}_{(1: \tilde{k} ; 1: \tilde{k})}>0$, take $x_{\tilde{k}+1 \tilde{k}+1}=\alpha_{\tilde{k}+1}$ to obtain $\mathbf{X}_{(1: \tilde{k}+1 ; 1: \tilde{k}+1)}>0$ and $\mathbf{Z}_{(1: \tilde{k}+1 ; 1: \tilde{k}+1)}>0$ for sufficiently large $\alpha_{\tilde{k}+1}>0$.

With the algorithm proposed above, a matrix $X>0$ can be computed such that $Z>0$ and Eqs. (13), (14) are satisfied. By specifying $y_{k}=x_{n n}$ and $y_{i}=-\left(x_{i+1 k}+[2+\mu(n-i)] x_{i k}\right), \quad i=$ $1,2, \ldots, n-1$, at the final step $(\tilde{k}=n)$, we arrive at $X>0$ and $Y$ satisfying equalities (13)-(16) and the inequality $X \operatorname{diag}(r)+\operatorname{diag}(r) X>0$.

The control law (11) proposed above admits a simple implementation provided that the initial condition $x(0)=x_{0} \in \mathbb{R}^{n}$ is known. Indeed, if a function $Q$ satisfies the conditions of Theorem 3 , then the equation $Q\left(V_{0}, x_{0}\right)=0$ possesses a unique positive solution $V_{0} \in \mathbb{R}_{+}$for an arbitrarily specified $x_{0} \in \mathbb{R}^{n}$. This solution can be found numerically, for example, by using the simplest dichotomy. On the other hand, for the closed-loop system (1), (11), the function $V$ considered 
as function of time can be found as the solution of the Cauchy problem $\dot{V}(t)=-V^{1-\mu}(t), t>0$, $V(0)=V_{0}$ (see proof of Theorem 4), hence, it admits the following closed-form representation:

$$
V(t)=\left(V_{0}^{\mu}-\mu t\right)^{1 / \mu}, \quad t \in\left[0, V_{0}^{\mu} / \mu\right] .
$$

Clearly, the linear dynamic feedback $u(\cdot)=w^{\top}(\cdot) x(\cdot)$ obtained in such a way (here, $w(\cdot)=$ $\left.V^{1-\mu}(\cdot) k D_{\mu}\left(V^{-1}(\cdot)\right)\right)$, satisfies constraints (3), (4).

In the sections to follow we propose an alternative scheme of implementation of the proposed control algorithm; it is motivated by robustness considerations.

\subsection{Suboptimal Finite-Time Controllers}

In order to solve the time-optimal control problem in the class of finite-time regulators, one has to minimize the quantity $T=V_{0}^{\mu} / \mu$ subject to the constraint $u(\cdot) \in \mathcal{U}$, where the control $u$ is defined by (11).

Corollary 3. Assume that the set of LMIs (10) together with

$$
\left(\begin{array}{cc}
1 & x_{0}^{\top} \\
x_{0} & D_{\mu}\left((\mu T)^{1 / \mu}\right) X D_{\mu}\left((\mu T)^{1 / \mu}\right)
\end{array}\right) \geq 0,
$$

where $X \in \mathbb{R}^{n \times n}$ and $y \in \mathbb{R}^{1 \times n}$, is feasible for some $\mu \in(0,1], x_{0} \in \mathbb{R}^{n} \backslash\{0\}$, and $T \in \mathbb{R}_{+}$.

Then the settling-time function of the closed-loop system (1), (11) satisfies the inequality $T_{0}\left(x_{0}\right) \leq$ $T$, where $x_{0} \in \mathbb{R}^{n}$ is given.

Proof. Denote $V_{0} \in \mathbb{R}_{+}: Q\left(V_{0}, x_{0}\right)=0$. Inequality (18) guarantees the fulfillment of the following relation: $Q\left((\mu T)^{1 / \mu}, x_{0}\right)=x_{0}^{\top} D_{\mu}\left(\frac{1}{(\mu T)^{1 / \mu}}\right) X^{-1} D_{\mu}\left(\frac{1}{(\mu T)^{1 / \mu}}\right) x_{0}-1 \leq 0=Q\left(V_{0}, x_{0}\right)$. Since $\frac{\partial Q(V, x)}{\partial V}<0$ for all $x \in \mathbb{R}^{n} \backslash\{0\}$, we have $V_{0} \leq(\mu T)^{1 / \mu}$, and by Theorem 4 we obtain $T_{0}\left(x_{0}\right)=\frac{V_{0}^{\mu}}{\mu} \leq T$.

The minimization of the parameter $T \in \mathbb{R}_{+}$subject to constraints (10), (18), yields the minimal settling time of the closed-loop system (1), (11) with initial condition $x(0)=x_{0}$. Without any constraints on the magnitude of the control input, the settling time $T_{0}\left(x_{0}\right)$ can be made arbitrarily small. Indeed, let the pair $\left(y_{0}, X_{0}\right)$ satisfy the matrix inequalities (10); then $y=\alpha y_{0}, X=\alpha X_{0}$ satisfy the same inequalities for any $\alpha \in \mathbb{R}_{+}$. Since $X_{0}>0$, for any $T>0$ and any $x_{0} \in \mathbb{R}^{n} \backslash\{0\}$ there exists $\alpha \in \mathbb{R}_{+}$such that inequality (18) is satisfied.

Corollary 4. Assume that the matrix inequalities (10), (18) together with

$$
\left(\begin{array}{cc}
X & y^{\top} \\
y & \frac{u_{0}^{2}}{(\mu T)^{\frac{2-2 \mu}{\mu}}}
\end{array}\right) \geq 0
$$

are feasible for some $\mu \in(0,1], T \in \mathbb{R}_{+}, x_{0} \in \mathbb{R}^{n}$, and $u_{0} \in \mathbb{R}_{+}$, where $X \in \mathbb{R}^{n \times n}$ and $y \in \mathbb{R}^{1 \times n}$.

Then the magnitude of the control input in the closed-loop system (1), (11) is bounded from above: $|u(V(x(t)), x(t))| \leq u_{0}$ for all $t \in\left[0, T_{0}\left(x_{0}\right)\right]$.

Proof. Using the Schur complement, Ineq. (19) writes $\frac{(\mu T)^{\frac{2-2 \mu}{\mu}}}{u_{0}^{2}} y^{\top} y \leq X$. Taking $X=P^{-1}$ and $y=k P^{-1}$ into account, we obtain $(\mu T)^{\frac{2-2 \mu}{\mu}} k^{\top} k \leq u_{0}^{2} P$, so that the inequality

$$
(\mu T)^{\frac{2-2 \mu}{\mu}} x^{\top} D_{\mu}\left(V^{-1}\right) k^{\top} k D_{\mu}\left(V^{-1}\right) x \leq u_{0}^{2} x^{\top} D_{\mu}\left(V^{-1}\right) P D_{\mu}\left(V^{-1}\right) x
$$


is valid for all $x \in \mathbb{R}^{n} \backslash\{0\} \quad V \in \mathbb{R}_{+}$. On the one hand we have

$$
\begin{gathered}
u^{2}(t)=V^{2-2 \mu}(t) x^{\top}(t) D_{\mu}\left(V^{-1}(t)\right) k^{\top} k D_{\mu}\left(V^{-1}(t)\right) x(t) \leq \\
\leq V_{0}^{2-2 \mu} x^{\top}(t) D_{\mu}\left(V^{-1}(t)\right) k^{\top} k D_{\mu}\left(V^{-1}(t)\right) x(t) \leq(\mu T)^{\frac{2-2 \mu}{\mu}} x^{\top} D_{\mu}\left(V^{-1}(t)\right) k^{\top} k D_{\mu}\left(V^{-1}(t)\right) x,
\end{gathered}
$$

since $V(x(t)) \leq V_{0}$ for $t \in\left[0, T\left(x_{0}\right)\right]$ (see (17)) and $T\left(x_{0}\right) \leq T$. On the other hand, the equality $Q(V, x)=0$ ensures

$$
u_{0}^{2} x^{\top} D_{\mu}\left(V^{-1}\right) P D_{\mu}\left(V^{-1}\right) x=u_{0}^{2} .
$$

Therefore, $u^{2}(V(t), x(t)) \leq u_{0}^{2}$ for $t \in\left[0, T\left(x_{0}\right)\right]$.

With the corollary above, a finite-time controller with a pre-specified upper bound on the magnitude of the control input can be designed. For $\mu=1$, Ineq. (19) no longer depends on $T$, so that the control input is globally bounded.

To summarize, the following finite-dimensional optimization scheme can be exploited to design a suboptimal finite-time regulator:

$$
\begin{gathered}
T \rightarrow \min _{X \in \mathbb{R}^{n}, y \in \mathbb{R}^{1 \times n}, \mu \in(0,1]} \\
\text { subject to }(10),(18),(19) .
\end{gathered}
$$

Note that in the scalar case $n=1$, the controller design procedure proposed above leads to the optimal solution of problem (2)-(3). Indeed, with $\mu=1$ and $n=1$, we have $V(x)=\sqrt{P}|x|$, $P \in \mathbb{R}^{1 \times 1}, x \in \mathbb{R}^{1}$, and $u_{\text {opt }}(x)=-u_{0} \operatorname{sign}[x]$.

For any fixed numbers $\mu \in(0,1]$ and $T \in \mathbb{R}_{+}$, the matrix inequalities (10), (18), (19) become linear and can be easily solved using standard software.

To solve this problem for a fixed $\mu$, an approach in [44] can be used, and the minimization with respect to the parameter $\mu$ can be accomplished via use of any known methods of scalar gradient-free optimization (for example, with the fminsearch routine in MATLAB).

The gap between the proposed suboptimal solution and the optimal one can hardly be evaluated analytically in the general case. By (12), the settling time depends on the Lyapunov function, which is specified implicitly and does not admit a closed-form representation in the majority of the problems.

\section{ROBUST REALIZATION OF SUBOPTIMAL FINITE-TIME CONTROLLERS}

As it was noted, the value of the Lyapunov function along the trajectory of the disturbance-free closed-loop system can be easily computed via the formula

$$
V(t)=\left(V_{0}^{\mu}-\mu t\right)^{1 / \mu}, \quad t \in\left[0, V_{0}^{\mu} / \mu\right],
$$

where $V_{0} \in \mathbb{R}_{+}: Q\left(V_{0}, x_{0}\right)=0$, and a suboptimal linear dynamic regulator can be specified by (11) for $V=V(t)$. However in practice, the original system (1) is subject to parametric uncertainties and exogenous disturbances; this often leads to considerable degradation of the performance of optimally designed control systems. Below we formulate two corollaries to Theorem 4 which will serve as the basis for the development of a practical realization scheme for finite-time regulators. These regulators will be designed in the form of a linear feedback with coefficients that change at discrete time instants.

Corollary 5. Let the finite-time regulator $u(V, x)$ be designed according to Theorem 4; then the control $u_{0}(x)=u\left(V_{0}, x\right)$ represents a stabilizing linear static regulator for system (1) for any fixed $V_{0} \in \mathbb{R}_{+}$. 
Proof. Rewrite the matrix inequality $A X+X A+b y+y^{\top} b^{\top}+\operatorname{diag}(r) P+P \operatorname{diag}(r)=0$ with $X=P^{-1}$ and $k=y X^{-1}$ in the form $P A+A^{\top} P+P b k+k^{\top} b^{\top} P+\operatorname{diag}(r) P+P \operatorname{diag}(r)=0$.

We then have

$$
\begin{gathered}
D_{r}\left(V_{0}^{-1}\right)\left(P A+A^{\top} P+P b k+k^{\top} b^{\top} P+\operatorname{diag}(r) P+P \operatorname{diag}(r)\right) D_{r}\left(V_{0}^{-1}\right)= \\
=D_{r}\left(V_{0}^{-1}\right) P D_{r}\left(V_{0}^{-1}\right) D_{r}^{-1}\left(V_{0}^{-1}\right) A D_{r}\left(V_{0}^{-1}\right)+D_{r}\left(V_{0}^{-1}\right) A^{\top} D_{r}^{-1}\left(V_{0}^{-1}\right) D_{r}\left(V_{0}^{-1}\right) P D_{r}\left(V_{0}^{-1}\right)+ \\
+D_{r}\left(V_{0}^{-1}\right) P D_{r}\left(V_{0}^{-1}\right) D_{r}^{-1}\left(V_{0}^{-1}\right) b k D_{r}\left(V_{0}^{-1}\right)+D_{r}\left(V_{0}^{-1}\right) k^{\top} b^{\top} D_{r}^{-1}\left(V_{0}^{-1}\right) D_{r}\left(V_{0}^{-1}\right) P D_{r}\left(V_{0}^{-1}\right)+ \\
+\operatorname{diag}(r) D_{r}\left(V_{0}^{-1}\right) P D_{r}\left(V_{0}^{-1}\right)+D_{r}\left(V_{0}^{-1}\right) P D_{r}\left(V_{0}^{-1}\right) \operatorname{diag}(r)=0 .
\end{gathered}
$$

Denoting $P_{0}=D_{r}\left(V_{0}^{-1}\right) P D_{r}\left(V_{0}^{-1}\right)>0$ and keeping in mind that $D_{r}^{-1}\left(V_{0}^{-1}\right) A D_{r}\left(V_{0}^{-1}\right)=V_{0}^{\mu} A$ and $D_{r}^{-1}\left(V_{0}^{-1}\right) b=V_{0} b$, we obtain

$$
V_{0}^{\mu}\left(P_{0} A+A^{\top} P_{0}\right)+P_{0} b V_{0} k D_{r}\left(V_{0}^{-1}\right)+D_{r}\left(V_{0}^{-1}\right) k^{\top} V_{0} b^{\top} P_{0}+\operatorname{diag}(r) P_{0}+P_{0} \operatorname{diag}(r)=0,
$$

or, equivalently,

$$
P_{0} A+A^{\top} P_{0}+P_{0} b k_{0}+k_{0}^{\top} b^{\top} P_{0}+\frac{1}{V_{0}^{\mu}}\left(\operatorname{diag}(r) P_{0}+P_{0} \operatorname{diag}(r)\right)=0,
$$

where $P_{0}>0$ and $k_{0}=V_{0}^{1-\mu} k D_{r}\left(V_{0}^{-1}\right)$.

Since $\operatorname{diag}(r) P+P \operatorname{diag}(r)>0$, we have $\operatorname{diag}(r) P_{0}+P_{0} \operatorname{diag}(r)>0$, which means that $u_{0}(x)=$ $u\left(V_{0}, x\right)=k_{0} x$ is a stabilizing linear static controller for system $(1)$, and $\tilde{V}(x)=x^{\top} P_{0} x$ is the corresponding Lyapunov function for the closed-loop system.

Corollary 6. Let $\left\{t_{i}\right\}_{i=0}^{\infty}, 0=t_{0}<t_{1}<t_{2}<\ldots$, be an arbitrary strictly increasing sequence of time instants and let the function $u(V, x)$ be defined according to Theorem 4.

Then the zero solution of system (1) with the piecewise-linear control

$$
u(x):=u\left(V_{i}, x\right) \quad \text { for } \quad t \in\left[t_{i}, t_{i+1}\right),
$$

where $V_{i}>0: Q\left(V_{i}, x\left(t_{i}\right)\right)=0$, is asymptotically stable.

Proof. I. We first consider the case $\lim _{i \rightarrow \infty} t_{i}=+\infty$. Let $V(\cdot)$ be a positive definite and radially unbounded Lyapunov function specified implicitly by the equation $Q(V, x)=0$. We prove that the sequence $\left\{V\left(x\left(t_{i}\right)\right)\right\}_{i=1}^{\infty}$ is monotonically decreasing and tends to zero. Clearly, this property ensures the convergence of the trajectory of the closed-loop system to the origin. Let us consider the segment $t \in\left[t_{i}, t_{i+1}\right)$ and the quadratic function $\tilde{V}_{i}(x)=x^{\top} P_{i} x$, where $P_{i}=D_{r}\left(V^{-1}\left(x\left(t_{i}\right)\right)\right) P D_{r}\left(V^{-1}\left(x\left(t_{i}\right)\right)>0\right.$. Over this segment, the control $u(x)$ has the form $u_{i}(x)=u\left(V\left(x\left(t_{i}\right)\right), x\right)=k_{i} x$ (see Corollary 5), where $k_{i}=V^{1-\mu}\left(x\left(t_{i}\right)\right) k D_{r}\left(V^{-1}\left(x\left(t_{i}\right)\right)\right)$. We thus obtain

$$
\frac{d \tilde{V}_{i}(x(t))}{d t}=x^{\top}(t)\left(P_{i} A+A^{\top} P_{i}+b k_{i}+k_{i}^{\top} b^{\top}\right) x(t), \quad t \in\left[t_{i}, t_{i+1}\right) .
$$

Similarly to the proof of Corollary 5 we have

$$
P_{i} A+A^{\top} P_{i}+b k_{i}+k_{i}^{\top} b^{\top}+\frac{1}{V\left(x\left(t_{i}\right)\right)}\left(\operatorname{diag}(r) P_{i}+\operatorname{diag}(r) P_{i}\right)=0 .
$$

Since $\operatorname{diag}(r) P_{i}+\operatorname{diag}(r) P_{i}>0$, there exists a number $\alpha \in \mathbb{R}_{+} \operatorname{such}$ that $\operatorname{diag}(r) P_{i}+\operatorname{diag}(r) P_{i} \geq$ $\alpha P_{i}$. Then $\frac{d \tilde{V}_{i}(x(t))}{d t} \leq-\frac{\alpha}{V\left(x\left(t_{i}\right)\right)} \tilde{V}_{i}(x(t))$ for $t \in\left[t_{i}, t_{i+1}\right)$. 
Hence, the function $\tilde{V}_{i}(x(t))$ exponentially decays along the trajectory of the closed-loop system $(1)$ on the time segment $\left[t_{i}, t_{i+1}\right)$; i.e., $\tilde{V}\left(x\left(t_{i+1}\right)\right) \leq e^{-\frac{\alpha}{V_{i}}\left(t_{i+1}-t_{i}\right)} \tilde{V}\left(x\left(t_{i}\right)\right)$. Therefore we have

$Q\left(V\left(t_{i}\right), x(t)\right)=x^{\top 1}(t) D_{r}\left(V^{-1}\left(x\left(t_{i}\right)\right)\right) P D_{r}\left(V^{-1}\left(x\left(t_{i}\right)\right)\right) x(t)-1=\tilde{V}_{i}(x(t))-1<$

$<\tilde{V}_{i}\left(x\left(t_{i}\right)\right)-1=Q\left(V_{i}\left(x\left(t_{i}\right)\right), x\left(t_{i}\right)\right)=0=Q(V(x(t)), x(t))$ for all $t \in\left(t_{i}, t_{i+1}\right]$.

For any given $x \in \mathbb{R}^{n} \backslash\{0\}$, the function $Q(V, x)$ is monotonically decreasing for all $V \in \mathbb{R}^{+}$(see Property $C_{4}$ in Theorem 3$)$. Hence, the inequality

$$
Q\left(V\left(x\left(t_{i}\right)\right), x(t)\right)<Q(V(x(t)), x(t)) \quad \forall t \in\left(t_{i}, t_{i+1}\right]
$$

implies $V(x(t))<V\left(x\left(t_{i}\right)\right)$ for all $t \in\left(t_{i}, t_{i+1}\right]$, i.e., the sequence $\left\{V\left(x\left(t_{i}\right)\right)_{i=1}^{\infty}\right.$ is monotonically decreasing. Moreover, the inequality $V(x(t)) \leq V(x(0))$ holds for all $t>0$, i.e., the sero solution of the closed-loop system is Lyapunov stable.

Since the function $V(x)$ is positive definite, the monotonically decreasing sequence $\left\{V\left(x\left(t_{i}\right)\right)\right\}_{i=1}^{\infty}$ tends to a limit. Let us show it is equal to zero. Assuming the converse, $\lim _{i \rightarrow \infty} V\left(x\left(t_{i}\right)\right)=V_{*}>0$, we obtain

$$
\forall \varepsilon>0 \quad \exists N=N(\varepsilon):\left|V\left(x\left(t_{i}\right)\right)-V_{*}\right|<\varepsilon \forall i \geq N .
$$

Since the control input $u(V, x)$ is a continuous function for all $x \in \mathbb{R}^{n}$ and all $V \in R_{+}$, we have $\left|u\left(V\left(x\left(t_{i}\right)\right), x\right)-u\left(V^{*}, x\right)\right|=\left|V^{1-\mu}\left(x\left(t_{i}\right)\right) k D_{r}\left(V^{-1}\left(x\left(t_{i}\right)\right)\right) x-\left(V_{*}\right)^{1-\mu} k D_{r}\left(V_{*}^{-1}\right) x\right| \leq \gamma(\varepsilon)\|x\| \quad \forall i \geq N$, where $\gamma(\cdot) \in \mathcal{K}$.

This means that for $t>t_{N}$, the closed-loop function can be written in the form $\dot{x}=A x+$ $b\left(k_{*} x+\delta(t) x\right)$, where $k_{*}=k\left(V_{*}\right)^{1-\mu} k D_{r}\left(V_{*}^{-1}\right)$ and $\|\delta\| \leq \gamma(\varepsilon)$. Corollary 5 guarantees that the matrix $A+b k_{*}$ is Hurwitz; then for a sufficiently small $\varepsilon>0$ the zero solution of this system is asymptotically stable. In turn, this means that $\lim _{i \rightarrow \infty} V\left(x\left(t_{i}\right)\right)=0$. The Lyapunov stability shown above implies asymptotic stability of the zero solution of the closed-loop system (1), (21).

II. If $\lim _{i \rightarrow \infty} t_{i}=t^{*}$, then for all $t>t^{*}$ we have $u(x)=u\left(V\left(t^{*}\right), x\right)$, and Corollary 5 ensures the desired asymptotic stability.

Corollary 6 guarantees that the discrete-time piecewise-linear realization of the finite-time regulator retains the stability of the closed-loop system independently of the sampling interval.

For a fixed $x_{i}=x\left(t_{i}\right), \quad i=1,2, \ldots$, the solution of the scalar equation $Q\left(V, x_{i}\right)=0$ can be found numerically; hence, in practice, the regulator thus obtained can be implemented with the use of a digital computing device (microcontroller) which changes the coefficients of the regulator at discrete time instants based on the current measurements. Recall that, by Property $C_{4}$ in Theorem 3 , we have $\frac{\partial Q}{\partial V}<0$ for all $x \in \mathbb{R}^{n} \backslash\{0\}$ and all $V \in \mathbb{R}_{+}$; i.e., the equation $Q\left(V, x_{i}\right)=0$ always has a unique solution with respect to $V \in \mathbb{R}_{+}$. This solution can be found by dichotomy or by the Newton method as $V^{[k+1]}=V^{[k]}-Q\left(V^{[k]}, x_{i}\right)\left[\frac{\partial Q\left(V^{[k]}, x_{i}\right)}{\partial V}\right]^{-1}, k=1,2, \ldots$, where $V^{[k]} \in \mathbb{R}_{+}$is the scalar variable, $x_{i} \in \mathbb{R}^{n}$ is a fixed vector, and $V^{[k]} \rightarrow V$ as $k \rightarrow \infty$.

To avoid infinitely large values of the coefficients of the regulator, the quantity $V$ is to be bounded from below by a certain parameter $V_{\min } \in \mathbb{R}_{+}$.

\section{A NUMERICAL EXAMPLE}

Let us consider system (1) for $n=2$ under the constraint $|u| \leq 1$. Discontinuous time-optimal regulator is known [5] to have the following form:

$$
u_{\text {opt }}\left(x_{1}, x_{2}\right)=-\operatorname{sign}\left(x_{2}+\sqrt{2\left|x_{1}\right|} \operatorname{sign}\left[x_{1}\right]\right) .
$$


The stabilizing finite-time regulator (11) obtained by solving the finite-dimensional optimization problem (20) for $\mu=1$ and $x_{0}=(1,0)$, has the following parameters:

$$
P=\left(\begin{array}{cc}
49.6139 & 18.8965 \\
18.8965 & 9.4482
\end{array}\right), k=\left(\begin{array}{ll}
-5.2511 & -3.0000
\end{array}\right) \text {. }
$$

Numerical simulation of the closed-loop model was performed by the explicit Euler method with fixed step, and the computational implementation of the finite-time regulator was performed by dichotomy.

The results of numerical experiments for the sampling interval $h=0.001$ and $V_{\min }=0.001$ are depicted in Fig. 1.

Fig. 1. Simulation results for $h=0.001$. 1: Optimal discontinuous regulator; 2: Suboptimal finite-time regulator.

As expected, the optimal discontinuous regulator demonstrates a better transient time; however the comparison of the algorithms for a bigger sampling time testifies to a higher robustness of the suboptimal finite-time regulator. Figure 2 depicts the results of simulations for $h=0.05$ and $V_{\min }=0.05$.

Fig. 2. Simulation results for $h=0.05$. 1: Optimal discontinuous regulator; 2: Suboptimal finite-time regulator.

Such a drawback of discontinuous time-optimal regulators is well know as the so-called chattering effect [23]. Chattering emerges in time-optimal systems exploiting sliding mode control [6]. The sampling time $h \geq 0.05$ is natural in many control applications, for instance, such as mobile robots. Indeed, in autonomous mobile systems, the computational resource is largely used for image processing, positioning, calculation of the trajectories of motion, etc., and it is only a small portion of it which is spent for producing control inputs.

\section{CONCLUSION}

In this paper we proposed a robust finite-time control design algorithm for plants described by chains of integrators; the implicit Lyapunov function method was adopted as the principal tool. The computational procedure for the minimization of the transient time in the closed-loop system was formulated as a semidefinite program. An algorithm for practical realization of the implicit finite-time regulator was also developed. The main drawback of the proposed control scheme is that it can be implemented only with the use of digital devices. However, keeping in mind the current level of electronics, this drawback does not seem to be crucial for the successful practical use of the proposed finite-time controllers. The main advantage of the approach, which is confirmed by numerical simulations, is a much higher degree of robustness as compared to time-optimal solutions implemented in the form of static state feedback.

\section{REFERENCES}

1. Feldbaum, A., Optimal Processes in Systems of Automatic Control. Autom. Remote Control, 1953, vol. 14 , no. 6 , pp. $71-728$.

2. Pontryagin, L.S., Boltyanski, V.G., Gamkrelidze, R.V., et al., The Mathematical Theory of Optimal Processes, New York: John Wiley \& Sons, 1962.

3. Bellman, R.E., Dynamic Programming, NJ: Princeton University Press, 1957. 
4. Lasserre, J.B., Prieur, C., and Henrion, D., Nonlinear Optimal Control: Numerical Approximations via Moments and LMI-Relaxations, Proc. 44th IEEE Conf. Decision Control, 2005, pp. 1648-1653.

5. Chernousko, F.L., Ananevskii, I.M., and Reshmin, S.A., Control of Nonlinear Dynamical Systems: Methods and Applications, Berlin: Springer-Verlag, 2008.

6. Dinuzzo, F. and Ferrara, A., Higher Order Sliding Mode Controllers with Optimal Reaching, IEEE Trans. Autom. Control, 2009, vol. 54, no. 9, pp. 2126-2136.

7. Boltyanski, V.G. and Poznyak, A.S., The Robust Maximum Principle: Theory and Applications, Boston: Birkhäuser, 2012.

8. Bernstein, D.S., Optimal Nonlinear, but Continuous, Feedback Control of Systems with Saturating Actuators, Int. J. Control, 1995, vol. 62, no. 5, pp. 1209-1216.

9. Glizer, V.Y. and Turetsky, V., Robust Controllability of Linear Systems, New York: Nova Science Publishers, 2012.

10. Bertsekas, D.P., Dynamic Programming and Suboptimal Control: A Survey from ADP to MPC, Eur. J. Control, 2005, vol. 11, pp. 310-334.

11. Bohl, A.H. and McAvoy, T.J., Linear Feedback vs. Time Optimal Control, Industrial and Engineering Chemistry Process Design and Development, 1976, vol. 15, no. 1, pp. 24-33.

12. Zubov, V.I., On Systems of Homogenous Differential Equations with Generalized Homogenous Righthand Sides, Izv. VUZov, Matematika, 1958, no. 1, pp. 80-88.

13. Rosier, L., Homogenous Lyapunov Function for Homogenous Coninuous Vector Field, Syst. Control Lett., 1992, vol. 19, pp. 467-473.

14. Bhat, S.P. and Bernstein, D.S., Geometric Homogeneity with Applications to Finite-time Stability. Math. Control Sig. Sys., 2005, vol. 17, pp. 101-127.

15. Bernuau, E., Efimov, D., Perruquetti, W., et al., On Homogeneity and Its Application in Sliding Mode Control, J. Franklin Inst., 2014, vol. 351, no. 4, pp. 1866-1901.

16. Adamy, J. and Flemming, A., Soft Variable-Structure Controls: A Survey. Automatica, 2004, vol. 40, pp. 1821-1844.

17. Polyakov, A., Efimov, D., and Perruquetti, W., Finite-time and Fixed-time Stabilization: Implicit Lyapunov Function Approach, Automatica, 2015, vol. 51, no. 1, pp. 332-340.

18. Courant, R. and John, F., Introduction to Calculus and Analysis, New York: Springer, 2000, vol. II, no. 1.

19. Korobov, V.I., Metod funktsii upravlyaemosti (Controllability Function Method), Moscow-Izhevsk: Regular and Chaotic Dynamics, 2007.

20. Boyd, S., El Ghaoui, L., Feron, E., et al. Linear Matrix Inequalities in System and Control Theory, Philadelphia: SIAM, 1994.

21. Polyak, B.T., Khlebnikov, M.V., and Shcherbakov, P.S., Upravlenie lineinymi sistemami pri vneshnikh vozmushcheniyakh: tekhnika lineinykh matrichnykh neravenstv (Control of Linear Systems Subject to Exogenous Disturbances: The Linear Matrix Inequalitiy Technique), Moscow: LENAND, 2014.

22. Poznyak, A., Polyakov, A., and Azhmyakov, V., Attractive Ellipsoids in Robust Control, Boston: Birkhäuser, 2014.

23. Utkin, V.I., Guldner, J., and Shi, J., Sliding Mode Control in Electro-Mechanical Systems, Boca Raton: CRC Press, 2009.

24. Biagiotti, L. and Zanasi, R., Time-Optimal Regulation of a Chain of Integrators with Saturated Input and Internal Variables: An Application to Trajectory Planning, Proc. 8th IFAC Symp. Nonlin. Control Syst., 2010, pp. 1278-1283.

25. Wang, X., Saberi, A., Stoorvogel, A., et al., Control of a Chain of Integrators Subject to Actuator Saturation and Disturbances, Int. J. Robust Nonlin. Control, 2012, vol. 22, no. 14, pp. 1562-1570. 
26. Control of Uncertain Systems with Bounded Inputs, Lect. Notes in Control Inform. Sci., S. Tarbouriech and G. Garcia, Eds., Springer Verlag, 1997.

27. Shinar, J., Glizer, V.Y., and Turetsky, V., Capture Zone of Linear Strategies in Interception Problems with Variable Structure Dynamics, J. Franklin Inst., 2014, vol. 351, no. 4, pp. 2378-2395.

28. Roxin, E., On Finite Stability in Control Systems, Rendiconti del Circolo Matematico di Palermo, 1966, vol. 15 , no. 3 , pp. 273-283.

29. Bhat, S.P. and Bernstein, D.S., Finite-time Stability of Continuous Autonomous Systems, SIAM J. Control Optimiz., 2000, vol. 38, no. 3, pp. 751-766.

30. Orlov, Y., Finite Time Stability and Robust Control Synthesis of Uncertain Switched Systems, SIAM J. Control Optimiz., 2005, vol. 43, no. 4, pp. 1253-1271.

31. Polyakov, A. and Poznyak, A., Method of Lyapunov Functions for Systems with Higher-order Sliding Modes, Autom. Remote Control, 2011, vol. 72, no. 5, pp. 944-963.

32. Polyakov, A. and Fridman, L., Stability Notions and Lyapunov Functions for Sliding Mode Control Systems, J. Franklin Inst., 2014, vol. 351, no. 4, pp. 1831-1865.

33. Zubov, V.I., Methods of A.M. Lyapunov and Their Applications, Leiden: Noordhoff, 1964.

34. Hahn, W., Stability of Motion, New York-Berlin-Heidelberg: Springer-Verlag, 1967.

35. Hermes, H., Nilpotent Approximations of Control Systems and Distributions, SIAM J. Control Optimiz., 1986, vol. 24, pp. 731-736.

36. Levant, A., Homogeneity Approach to High-order Sliding Mode Design, Automatica, 2005, vol. 41, pp. 823-830.

37. Ryan, E.P., Universal Stabilization of a Class of Nonlinear Systems with Homogeneous Vector Fields, Syst. Control Lett., 1995, vol. 26, pp. 177-184.

38. Hong, Y., $H_{\infty}$ Control, Stabilization, and Input-output Stability of Nonlinear Systems with Homogeneous Properties, Automatica, 2001, vol. 37, no. 7, pp. 819-829.

39. Bernuau, E., Polyakov, A., Efimov, D., et al., Verification of ISS, iISS and IOSS Properties Applying Weighted Homogeneity, Syst. Control Lett., 2013, vol. 62, no. 12, pp. 1159-1167.

40. Bacciotti, A. and Rosier, L., Lyapunov Functions and Stability in Control Theory, 2nd edition, Springer, 2005.

41. Sontag, E.D., Smooth Stabilization Implies Coprime Factorization, IEEE Trans. Autom. Control, 1989, vol. 34, no. 4, pp. 435-443.

42. Dashkovskiy, S.N., Efimov, D.V., and Sontag, E.D., Input to State Stability and Allied system Properties Autom. Remote Control, 2011, vol. 72, no. 8, pp. 1579-1614.

43. Korobov, V.I., A General Approach to Synthesis Problem, Doklady AN SSSR, 1979, vol. 248, pp. 10511063.

44. Tuan, H.D., Apkaryan, P., and Tuy, H., Advanced Global Optimization Algorithms for Parameterized LMIs, Proc. 38th Conf. Decision Control, Pheonix, 1999. 


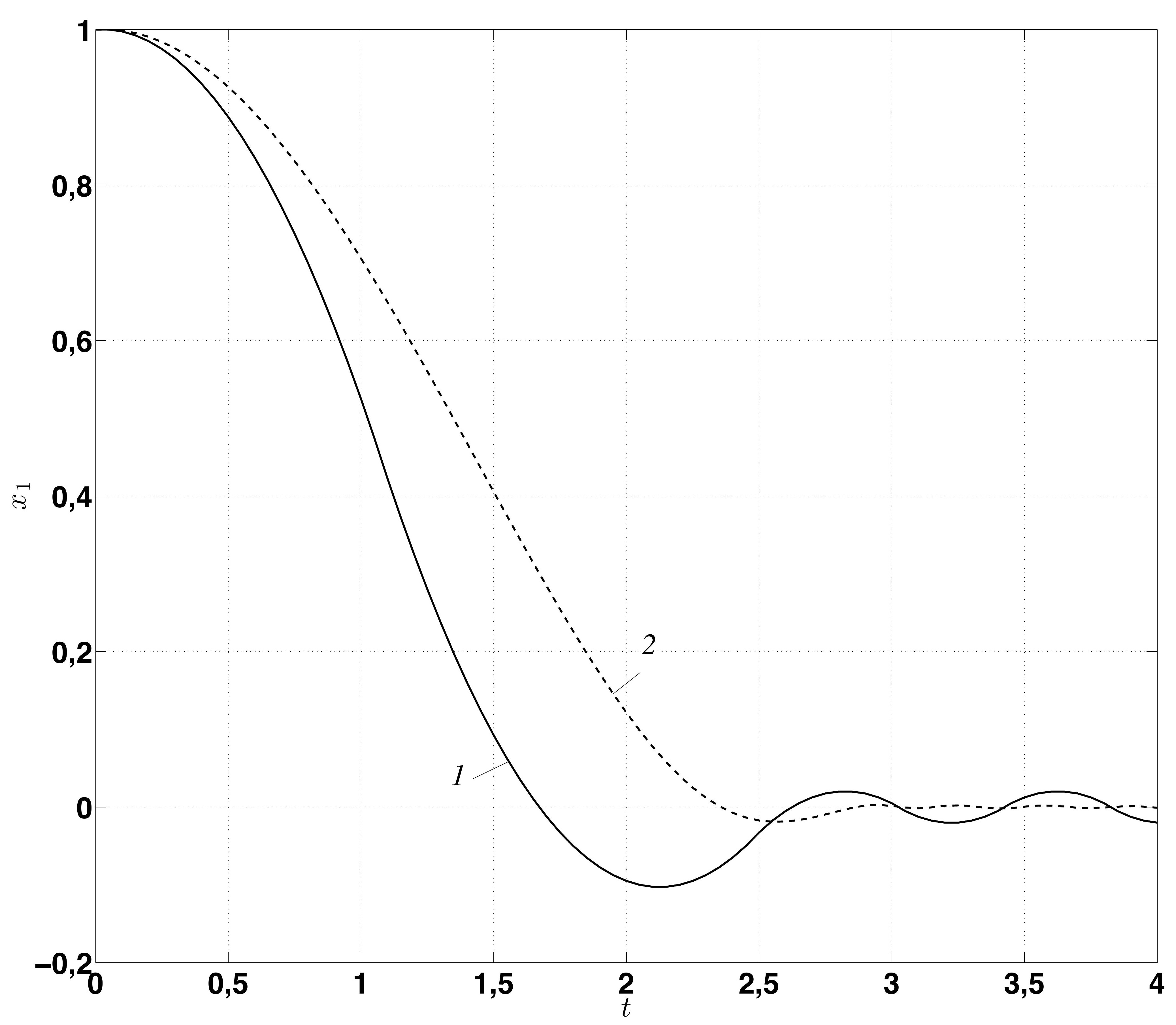

